\title{
A Simple Derivation of the Acoustic Boundary Condition in the Presence of Flow
}

\author{
F. Farassat \\ NASA Langley Research Center \\ Hampton, Virginia 23681
}

M.H. Dunn

Old Dominion University

Norfolk, Virginia 23508

In modeling the noise propagation in a duct of a ducted fan engine, the authors needed the boundary condition satisfied by the acoustic pressure on a lined duct wall in the presence of flow. The result in the form used by the authors was derived by M.K. Myers [1] who presented a formal derivation of a related result by K. Taylor [2]. A good review of the subject can be found in reference 3. Here a brief and simple derivation of the acoustic boundary condition of Myers is given. The main difference in the derivation from that in reference [1] is that the Gaussian coordinate system $\left(q^{I}, q^{2}, q^{3}\left(q^{l}, q^{2}, t\right)\right)$ is used to specify the instantaneous position, i.e. Lagrangian variable, of the point on the mean position of the wall with curvilinear (Gaussian) coordinates $\left(q^{l}, q^{2}\right)$. Myers uses a locally orthogonal coordinate system which is somewhat less specific than what is used here.

One assumes that one has a base or background flow with velocity $\vec{u}_{\theta}(\vec{x})$ which is time independent perturbed by a small velocity distribution $\varepsilon \vec{u}_{l}(\vec{x}, t)$ where $0<\varepsilon<<1$. One also assumes that the wall boundary's mean position $S_{0}$ is independent of time and specified by the position vector $\vec{x}_{0}\left(q^{1}, q^{2}\right)$ where $\left(q^{1}, q^{2}\right)$ are the curvilinear (Gaussian) coordinates on the boundary surface. The position of the time dependent boundary $S$ is given by 


$$
\vec{x}\left(q^{l}, q^{2}, q^{3}, t\right)=\vec{x}_{t}\left(q^{l}, q^{2}\right)+\varepsilon q^{3}\left(q^{l}, q^{2}, t\right) \vec{n}_{o}\left(q^{l}, q^{2}\right),
$$

where $\vec{n}_{0}$ is the local unit normal to $S_{0}$ and $\varepsilon q^{3}$ is the distance along the normal $\vec{n}_{0}$ from $S_{0}$ to $S$ at $\left(q^{l}, q^{2}, t\right)$. The fundamental physical requirement at the boundary is

$$
\left(\vec{u}_{0}+\varepsilon \vec{u}_{l}\right) \cdot \vec{n}_{0}=\varepsilon \frac{\partial q^{3}}{\partial t}
$$

This is the instantaneous equality of the normal fluid velocity and the surface velocity. Note that the symbol $\varepsilon$ will be retained for order of magnitude comparison for now.

The left side of equation (2) will now be expanded as follows and equated to the right side:

$$
\begin{aligned}
& {\left[\vec{u}_{0}\left(\vec{x}_{\theta}+\varepsilon q^{3} \vec{n}_{0}\right)+\varepsilon \vec{u}_{l}\left(\vec{x}_{0}+\varepsilon q^{3} \vec{n}_{0}\right)\right] \cdot \vec{n}_{0}} \\
& =\vec{u}_{0}\left(\vec{x}_{0}\right) \cdot \vec{n}_{0}+\varepsilon\left[q^{3} \vec{n}_{0} \cdot \nabla \vec{u}_{0}\left(\vec{x}_{\theta}\right)+\vec{u}_{l}\left(\vec{x}_{\theta}\right)\right] \cdot \vec{n}_{0}=\varepsilon \frac{\partial q^{3}}{\partial t} .
\end{aligned}
$$

The equality of the zeroth and first order terms from both sides gives

$$
\vec{u}_{0}\left(\vec{x}_{\theta}\right) \cdot \vec{n}_{0}=0
$$

and

$$
q^{3} \vec{n}_{0} \cdot\left[\vec{n}_{0} \cdot \nabla \vec{u}_{0}\left(\vec{x}_{0}\right)\right]+\vec{u}_{l}\left(\vec{x}_{\theta}\right) \cdot \vec{n}_{0}=\frac{\partial q^{3}}{\partial t}
$$

Equation (4) tells us that the normal velocity based on the mean flow is zero on the mean surface. Equation (5) can be written as

$$
\vec{u}_{l}\left(\vec{x}_{\theta}\right) \cdot \vec{n}_{0}=\frac{\partial q^{3}}{\partial t}-q^{3} \vec{n}_{\theta} \cdot\left[\vec{n}_{\theta} \cdot \nabla \vec{u}_{0}\left(\vec{x}_{\theta}\right)\right]
$$

One now assumes $\varepsilon=1$ and thus $\left|\vec{u}_{1}\right|<<\left|\vec{u}_{\theta}\right|$ and $q^{3}$ is the local normal distance between $S_{0}$ and $S$ as a function of time. Note that $\partial q^{3} / \partial t$ is the local normal velocity of $S$ in terms of the Lagrangian variables $\left(q^{l}, q^{2}\right)$. Using Eulerian variables $\vec{x}_{0}$, one can define a new function $g\left(\vec{x}_{0}, t\right)$ such that 
$q^{3}=g\left(\vec{x}_{f}, t\right)$. Note that since $q^{3}$ is the normal distance between $S_{0}$ and $S$, one has $|\nabla g|=1$. One notes that

$$
\frac{\partial q^{3}}{\partial t}\left(q^{l}, q^{2}\right)=\frac{\partial g}{\partial t}+\vec{u}_{0}\left(\vec{x}_{0}\right) \cdot \nabla g
$$

Using this result in equation (6) gives equation (11) of Myers [1]:

$$
\vec{u}_{j}\left(\vec{x}_{0}\right) \cdot \vec{n}_{0}=\frac{\partial g}{\partial t}+\vec{u}_{0}\left(\vec{x}_{0}\right) \cdot \nabla g-g \vec{n}_{0} \cdot\left[\vec{n}_{0} \cdot \nabla \vec{u}_{0}\left(\vec{x}_{0}\right)\right]
$$

which is the condition that the perturbation velocity $\vec{u}_{l}$ must satisfy on the mean surface $S_{0}$.

One now derives the liner boundary condition based on equation (8). For a time harmonic disturbance proportional to $e^{i \omega t}$, the complex acoustic pressure $p$ and $g$ are related to each other by the following relation on $S_{0}$ :

$$
g=-\frac{p}{i \omega Z}
$$

where $Z$ is the complex normal impedance. Using this result in equation (8) gives

$$
\vec{u}_{l} \cdot \vec{n}_{0}=-\frac{p}{Z}-\frac{1}{i \omega} \vec{u}_{0} \cdot \nabla\left(\frac{p}{Z}\right)+\frac{p}{i \omega Z} \vec{n}_{0} \cdot\left(\vec{n}_{0} \cdot \nabla \vec{u}_{0}\right),
$$

which is the liner boundary condition, equation (15), in Myers [1]. Equation (10) is implemented in a ducted fan noise prediction code developed for NASA Langley Research Center by the authors [4].

\section{References}

1. M.K. Myers 1980 Journal of Sound and Vibration 71, 429-434. On the Acoustic Boundary Condition in the Presence of Flow.

2. K. Taylor 1979 Journal of Sound and Vibration 65, 125-136. Acoustic Generation by Vibrating Bodies in Homentropic Potential Flow at Low Mach Number. 
3. A. Neyfeh, J. Kaiser and D. Telionis 1975 ALAA Journal 13, 130-153. Acoustics of Aircraft EngineDuct Systems.

4. M.H. Dunn, J. Tweed, and F.Farassat, 1999 Journal of Sound and Vibration (in press). The Application of a Boundary Integral Equation Method to the Prediction of Ducted Fan Engine Noise. 